Case 4.-Miss H., aged 16 , a schoolgirl, came to be examined for glasses as her head had been aching for a week. Sinusitis was diagnosed; three treatments with vacuum pump effected a cure.

CASE 5.-Mr. H., aged 40, electrician, had previously been fitted with glasses. He has an extreme deviation of the septum to the left completely blocking the left nostril, and during the past year he twice set date for submucous operation. He complained of photophobia and pain in and over the left eye, radiating to the occiput. The pupil was contracted; there was no nasal discharge, and headache was not particularly increased by leaning forward. The pain was severe enough to awaken the patient. The pupil was dilated and he was sent home. On the following day there was no relief of symptoms, but the patient was relieved at once by application of the vacuum-pump, and there was no return of symptoms after the fourth treatment. The pump certainly corrected this diagnosis and as I look back I can recall many cases that would have been cleared up by this little instrument.

Case 6.-Miss H., aged 25, has had progressive deafness with tinnitis and fuliess of the ears for two years. There was partial stenosis of the nasal passage, which was relieved by operative means, and then infations employed with benefit up to a certain point. After inflation, the patient would soon complain of stuffiness and noise. The vacuum-pump has started this patient on the road to recovery, as the serous secretion is now sucked from the middle ear; the sense of stultiness and noise rarely returus and the liearing is now steadily improving, while before it was at a standstill.

There are great possibilities in these cases in which there is hypersecretion of the middle-ear and its accessory cavities. Many physicians are undoubtedly using the vacuum-pump, but in $m y$ clinical wandering $I$ have not come across them. Future reports along these lines will be interesting.

\section{A CASE OF PRIMITIVE OBSTETRICS}

\section{WEINSTOCK, M.D. GERMaNTOWN, PA.}

History.-On Jan. 1, 1911, at 1:30 p. m.. I was called to a negress, ii-para, age 22 , unmarried, a domestic in a boarding house who had given birth to a boy at $3: 30 \mathrm{a} . \mathrm{m}$. of the same day under the following circumstances: Thinking herself pregnant for but seven months, she had made no arrangements for the birth of this child, as she had had twins about a year and a half ago, both children dying soon after birth. On Dec. 31 , 1910 , at 11 p. m., while alone in the house, she experienced a sudden pain in the abdomen which made her "double up." It soon passed off, and she thought it was simply cramp. In a short time pains began again, and she suspected that they were labor pains. After four and a half hours a boy was born. She waited till the placenta was born, and with seissors eut the cord about six inches from the umbilicus, and did not tie the cord which was still attached to the chilk. Washing herself as well as she could, and wrapping the child up in a blanket, she went to sleep. Next morning at 6:30 a. $\mathrm{m}$. she rose, dressed, prepared breakfast for the family, did her other work, and then told her mistress what had happened early in the morning. She then wrapped all her bloody linen around the placenta, placed the bundle in a suit case, wrapped the child in some blankets, and went home on font, a distance of six squares. Reaching home, which by the way was the home of the father, she went to bed and called me.

Examination.-I found the patient to be a fairly well-devel. oped young woman, of apparent good health. Temperature was 98.6 F., pulse 65, respiration 18 . Vaginal examination showed a slight median tear of the perineum, but the uterus was in normal condition following delivery. She was given a uterine douche. The child was fully developed, about 8 pounds in weight, and showel no marks or deformities. After giving the child a bath. I tied the cord about one and a half inches from the umbilicus, and cut off the rest. This pro. cedure was, I think, not absolutely necessary, as the cord was absolutely bloodless, but was done for sake of precaution.

Subsequent History.- The mother never had a temperature above $98.8 \mathrm{~F}$., or a pulse rate above 70 during her six days in bed. She got out of the bed on Jan. 6, 1911, and went back to her position. The child is doing well, and seems in no way concerned as to the attention he received when he entered this world.

The long time the child was attached to the placenta, a period, so far as the mother can guess, of ten minutes, probably saved it from hemorrhage. This case seems to emphasize the point that in a normal case the less the attending physician does the better it will be for the mother.

44 West Chelten Avenue.

\section{A METHOD OF STAINING GRANULAR CASTS AND OTHER TUBE PRODUCTS}

\section{H. B. ERDMAN, M.D. WERTERSVILLE, PA.}

Since it is often desirable to stain and mount tuberasts for preservation, the following method is suggesterl as being simple and easily carried out, and at the same time showing distinctly many features difficult to recognize in the unstained specimen:

Sediment the fresh urine in a centrifuge: pour off the supernatant urine, and fill the tube with 0.4 per cent. sodium chlorid solution. Gently shake the deposit, centrifuge, pour ofi the solution, and repeat the washing, this time centrifuginy tiil the sediment forms a dense clump at the bottom of the tube.

Slowly pour off the solution, invert the tube and allow it to drain for a few moments; the sediment will remain in the point of the tube.

With a long pipette transfer a small drop of sediment to a cover-glass, gently spread with a fine platinum wire loop. and dry in air. The spread must be thin.

Treat for three minutes with a 5 per cent. aqueous solution of mercuric chlorid. Wash thoroughly in water.

Stain for five minutes with a fresh mixture of equal parts of the following solutions: methylene blue, 0.3 per cent. aqueous solution; fuchsin, 0.02 per cent. aqueous solution: wash in water, dry, and mount in balsam.

To demonstrate fat. stain the fixed spread for five minutes with a solution of Sudan III in 70 per cent. slcohol. Wash in water, and apply the double stain as before. Mount in glycerin or glycerin jelly.

Hucin and chromatin stain violet to indigo, granular casts and protoplasm stain from pink to dark red, waxy casts stain bright red and fat globules bright orange.

One washing may suffice when the urine contains a very small amount of sediment. The stains may be used singly, first staining with fuchsin. washing in water and counter-staining with methylene blue.

Leukocytes and epithelial cells stain distinctly, the protoplasm of renal epithelium usually taking a dark red color, that of squamous epithelium a light pink. Red blood-cells may show a pale salmon color. Mucinous products are distinguished chiefly as filaments, agglutinations, showing their structure by longitudinal or spiral striations, and homogeneous casts. These forms merge into each other, there are no sharp dividing lines. They are probably not of much significance when not accompanied by granular casts. 